\title{
605.
}

\section{NOTE ON A POINT IN THE THEORY OF ATTRACTION.}

[From the Proceedings of the London Mathematical Society, vol. vi. (1874-1875), pp. 79-81. Read February 11, 1875.]

CONSIDER a mass of matter distributed in any manner on a surface, and attracting points $P, Q$ not on the surface. Consider a point $Q$ accessible from $P$, viz. such that we can pass continuously from $P$ to $Q$ without passing through the surface. (It is hardly necessary to remark that, if for example the matter is distributed over a hemisphere or segment of a closed surface, then by the surface we mean the hemisphere or segment, not the whole closed surface.) The potential and its differential coefficients ad infinitum, in regard to the coordinates of the attracted point, all vary continuously as we pass from $P$ to $Q$; and it follows that the potential is one and the same analytical function of $(a, b, c)$, the coordinates of the attracted point, for the whole series of points accessible from the original point $P$; in particular, if the surface be an unclosed surface, for instance a hemisphere or segment of a sphere, then every point $Q$ whatever not on the surface is accessible from $P$; and the theorem is that the potential is one and the same analytical function of $(a, b,-c)$, the coordinates of the attracted point, for any position whatever of this point (not being a point on the surface). But this seems to give rise to a difficulty. Consider the matter as uniformly distributed over a closed surface, and divide the closed surface into two segments: the potential of the whole shell is the sum of the potentials of the two segments; and the potential of the first segment being always one and the same function of $(a, b, c)$, whatever may be the position of the attracted point, and similarly the potential of the second segment being always one and the same function of $(a, b, c)$, whatever may be the position of the attracted point; then the potential of the whole shell is one and the same function of $(a, b, c)$, whatever may be the position of the attracted point. This we know is not the case for a uniform spherical 
shell; for the potential is a different function for external and interior points, viz. for internal points it is a constant, $=M \div$ radius; for external points it is $=\frac{M}{\sqrt{a^{2}+b^{2}+c^{2}}}$, if $a, b, c$ are the coordinates measured from the centre of the sphere.

The difficulty is rather apparent than real. Reverting to the case of an unclosed surface or segment, and considering the continuous curve from $P$ to $Q$, let this be completed by a curve from $Q$ to $P$ through the segment; viz. we thus have $P, Q$ points on a closed curve or circuit meeting the segment in a single point. To fix the ideas, the circuit may be taken to be a plane curve, and the position of a point on the circuit may be determined by means of its distance $s$ from a fixed point on the circuit. Considering this circuit as drawn on a cylinder, we may at each point of the circuit measure off, say upwards, along the generating line of the cylinder, a length or ordinate $z$, proportional to the potential of the point on the circuit, the extremities of these distances forming a curve on the cylinder, say the potential curve. We may draw a figure representing this curve only; the points $P, Q$ being marked

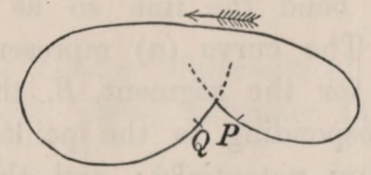

as if they were points on the curve (viz. at the upper instead of the lower extremities of the corresponding ordinates $z$ ): the generating lines of the cylinder, and the plane section which is the circuit, not being shown in the figure. The potential curve is then, as shown in the figure, a continuous curve, viz. we pass from $P$ to $Q$ in the direction of the arrow, or along that part of the circuit which does not meet the segment, a curve without any abrupt change in the value of the ordinate $z$ or of any of its differential coefficients, $\frac{d z}{d s}, \frac{d^{2} z}{d s^{2}}$, \&c.; but there is, corresponding to the point where the circuit meets the surface, an abrupt change in the direction of the potential curve or value of the differential coefficient $\frac{d z}{d s}$, viz. the point on the curve is really a node, the two branches crossing at an angle, as shown by the dotted lines, but without any potentials corresponding to these dotted lines.

In the case of two segments forming a closed surface, or say two segments forming a complete spherical shell; then, if the points $P, Q$ are one of them internal, the other external, the circuit, assuming it to meet the first segment in one point only, will meet the second segment in at least one point; the potential curves corresponding to the two segments respectively will have each of them, at the point corresponding to the intersection of the circuit with the segment, a node; and it hence appears how, in the potential curve corresponding to the whole shell (for which curve the ordinate $z$ is the sum of the ordinates belonging to the two segments respectively), there will be a discontinuity of form corresponding to the passage from an exterior to an interior point.

C. IX. 
This is best shown by the annexed figure, which represents a uniform spherical shell made up of two segments, one of which is taken to be a small segment or disc having the point $A$ for its centre, the other the large segment $B$, which is the remainder of the shell; the circuit is taken to be the right line ..PAQB.. through

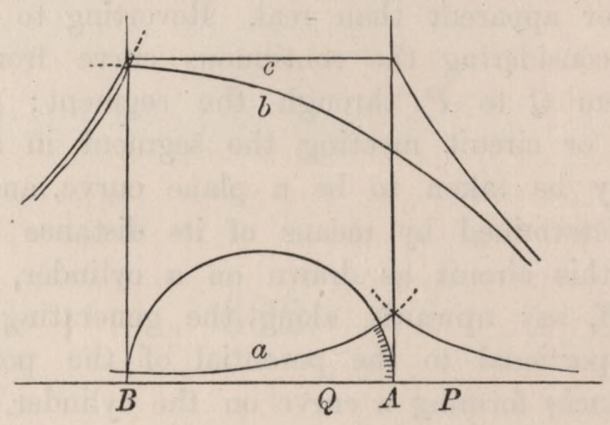

the centre of the sphere (viz. we may imagine the two extremities meeting at infinity, or we may, outside the sphere, bend the line so as to unite the two extremities, thus forming a closed curve). The curve $(a)$ represents the potential curve for the segment $A$, the curve $(b)$ that for the segment $B$, these two curves having, as shown by the dotted lines, nodes corresponding to the points $A, B$ respectively (but these dotted portions not indicating any potentials); and then, drawing at each point the ordinate which is the sum of those for the curves $(a),(b)$ respectively, we have the discontinuous curve (c), composed of a horizontal portion and two hyperbolic branches, which is the potential curve for the whole spherical shell.

Practically the figure is constructed by drawing the curves $(c),(a)$, and from them deducing the curve (b). As regards the curve (a) it may be noticed that, treating the segment $(a)$ as a plane disc, the curve $(a)$ is made up of portions of two hyperbolas; viz. it breaks up into two curves, instead of being, as assumed in the discussion, a single curve; this is a mere accident, not affecting the theory; and, in fact, taking the segment to be what it really is, the segment of a sphere the potential curve does not thus break up. 